\title{
EVIDENCE FOR ALLOY SPLITTING OF Ge RELATED DX STATE IN $\mathrm{Al}_{x} \mathrm{Ga}_{1-x} \mathrm{As}$
}

\author{
C. Skierbiszewski, R. Piotrzkowski
}

High Pressure Research Center, Polish Academy of Sciences

Sokołowska 29, 01-142 Warsaw, Poland

AND K. LUBKE

Institut für Experimentalphysik, Johannes-Kepler-Universität, 4040 Linz, Austria

Thermal emission from resonant DX levels in Ge-doped GaAlAs was studied by measuring the temperature transients of free electron concentration. Pressure was used to fill the levels with electrons. Two emission peaks are observed in AlGaAs:Ge. This enables us to confirm that $\mathrm{Ge}$, similarly to Si dopant, is stabilized upon dangling bond $C_{3 v}$ configuration in AlGaAs. Analysis of experimental data allows us to determine parameters of two components of the DX multilevel system. Evaluated alloy splitting of ground and top of the barrier states: $45 \mathrm{meV}$ is comparable with determined for Si donor.

PACS numbers: 71.55.-i, 72.20.Fr

\section{Introduction}

It was established both experimentally and theoretically that in addition to shallow state, Ge in GaAs and AlGaAs creates two deep, localized states: metastable, two-electron DX state and one electron state with $A_{1}$ symmetry $[1,2]$. Up to now, the microscopic picture of two-electron bonding DX state is still subject of controversy. Schmidt et al. [3] basing on ab initio pseudopotential calculations suggest that behavior of the $\mathrm{Ge}_{\mathrm{Ga}}$ defect is qualitatively different from the broken-bond model usually associated to $\mathrm{Si}_{\mathrm{Ga}}[4,5]$ : for the Ge impurity, a negative- $U$ behavior is found for breathing-mode relaxations of the Ge neighbors, in $T_{d}$ symmetry.

In this paper we investigate the emission from Ge-related DX state. If this state is stabilized upon broken-bond geometry, then pronounced differences between emission energies (associated with different local surroundings of Ge atoms in $\mathrm{AlGaAs}$ ) is expected. Such effect, originating from second neighbors, was observed and studied in detail for Si-related DX states in AlGaAs [6]. 


\section{Experiment and data analysis}

Emission from the $\mathrm{Al}_{0.1} \mathrm{Ga}_{0.9} \mathrm{As}$ samples doped with $\mathrm{Ge}$ on the level $4 \times 10^{18} \mathrm{~cm}^{-3}$ was investigated. We analyzed emission of electrons from DX states after high pressure freeze-out (HPFO) of electrons on these states [6]. HPFO is an unique tool which enabled us to create a nonequilibrium population of DX at low temperature. In the HPFO cycle, pressure is applied at $300 \mathrm{~K}$ allowing to populate the DX states by electrons. Then sample is cooled down with pressure below the metastability temperature $T_{M}$ and then pressure is released. Electrons remain then on the DX states and when temperature is rised the emission from those states can be observed.

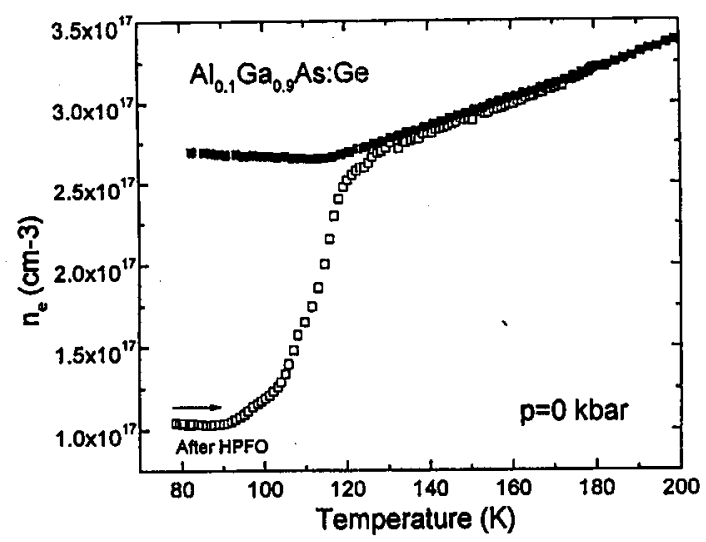

Fig. 1. Temperature dependence of electron concentration at ambient pressure without (full squares) and after high pressure freeze-out (empty squares).

Figure 1 shows dependence of electron concentration at ambient pressure vs. temperature without and after HPFO. Analysis of emission after the HPFO is presented in Fig. 2, where $\mathrm{d} n_{\mathrm{e}} / \mathrm{d} t$ vs. $1000 / T$ is plotted. Within experimental accuracy at least two peaks are visible - resembling emission from different DX levels. One peak is much bigger, which means that almost all of the electrons are populating only one level.

In general, the emission from the DX state is given by the equation

$$
\frac{-\mathrm{d} N_{\mathrm{DX}}}{\mathrm{d} t}=e_{\mathrm{DX}}^{\infty} \exp (-\eta x) N_{\mathrm{DX}}
$$

where $x=1 / T, \eta=\Delta E_{\mathrm{em}} / k_{\mathrm{B}}$.

Here we present approach, in which analysis of emission becomes simple. Under assumption that $1 / T$ is a linear function of time $t: 1 / T=c t$, which can be easily realized in experimental setup, the change of the electron concentration $\mathrm{d} n_{\mathrm{e}} / \mathrm{d} t\left(-\mathrm{d} N_{\mathrm{Dx}} / \mathrm{d} t=\mathrm{d} n_{\mathrm{e}} / \mathrm{d} t\right)$ can be expressed in the following manner [7]:

$$
\frac{\mathrm{d} n_{\mathrm{e}}}{\mathrm{d} t}=y_{\max } \exp \left\{-\eta\left(x-x_{\max }\right)-\exp \left[-\eta\left(x-x_{\max }\right)\right]+1\right\}
$$

where $y_{\max }=\eta|c| N_{\mathrm{DX}}\left(t_{0}\right) \exp \left\{\exp \left[-\eta x\left(t_{0}\right)\right]-1\right\}, \exp \left(-\eta x_{\max }\right)=\eta|c| / e_{\mathrm{DX}}^{\infty}$, here $t_{0}$ is an initial time for measurements. 


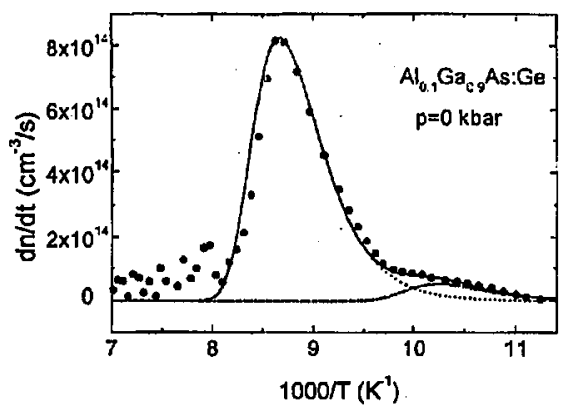

Fig. 2. Derivative of electron concentration over the time, $\mathrm{d} n_{\mathrm{e}} / \mathrm{d} t$, as a function of inverse temperature 1000/T. Points - experimental data. Dashed lines - fittings of emission energies from alloy splitted DX states. Solid line - total emission taken as a sum of individual fittings.

Equation (2) describes situation for emission from one DX state. Emission from each DX state can be thus modeled separately by Eq. (2) (when broadening is smaller than peak separation). By fitting two peaks (see Fig. 2) we can get two emission energies: $\Delta E_{\mathrm{em} 1}=215 \mathrm{meV}$ and $\Delta E_{\mathrm{em} 2}=260 \mathrm{meV}$, as well as pre-exponential coefficient $e_{\mathrm{DX}}^{\infty}=2 \times 10^{9} \mathrm{~s}^{-1}$ which is the same for both DX states.

\section{Conclusions}

Two emission peaks are observed in AlGaAs:Ge. This enables us to confirm that Ge-related DX states, similarly to Si ones, are stabilized upon dangling bond $C_{3 v}$ configuration in agreement with Chadi and Chang model [5]. The analysis of experimental data allows us to determine parameters of two components of the DX multilevel system. Evaluated alloy splitting of ground and top of the barrier states: $45 \mathrm{meV}$ is comparable with determined for $\mathrm{Si}$ donor.

\section{Acknowledgment}

Work supported by the Fonds zur Förderung der Wissentschaftlichen Forschung, Austria.

\section{References}

[1] C. Skierbiszewski, T. Suski, P. Wisniewski, W. Jantsch, G. Ostermayer, Z. Wilamowski, P.G. Walker, N.J. Mason, J. Singleton, Appl. Phys. Lett. 63, 3209 (1993).

[2] C. Skierbiszewski, P. Wisniewski, T. Suski, Z. Wilamowski, G. Ostermayer, W. Jantsch, P. Walker, N.J. Mason, J. Singleton, Mater. Sci. Forum 143-147, 1013 (1994).

[3] T.M. Schmidt, A. Fazzio, M.J. Caldas, Phys. Rev. B 53, 1315 (1996).

[4] J. Dabrowski, M. Scheffler, Mater. Sci. Forum 83-87, 735 (1992).

[5] D.J. Chadi, K.J. Chang, Phys. Rev. Lett. 61, 873 (1988).

[6] R. Piotrzkowski, E. Litwin-Staszewska, P. Lorenzini, J.L. Robert, Semicond. Sci. Technol. 7, 103 (1992).

[7] R. Piotrzkowski, E. Litwin-Staszewska, T. Suski, L. Konczewicz, J.L. Robert, W. Stankiewicz, J. Appl. Phys. 73, 2572 (1993). 